\title{
LETTER OPEN \\ Real-world evidence for improved outcomes with histamine antagonists and aspirin in 22,560 COVID-19 patients
}

Signal Transduction and Targeted Therapy (2021)6:267

\section{Dear Editor,}

The COVID-19 pandemic has driven great interest in the therapeutic potential of repurposed drugs with well-established benefits and safety profiles (toxicity, bioavailability, etc.), many of which act via signal transduction pathways. One category of such drugs is those that reduce acid production in gastroenterological contexts. Acid-suppressing drugs belong to two main classes, based on their mechanisms of action: (i) proton-pump inhibitors (PPIs) sterically block $\mathrm{H}^{+} / \mathrm{K}^{+}$-ATPase pumps, impeding the final step of acid release in the gastric mucosa. (ii) Histamine $\mathrm{H}_{2}$ receptor antagonists ( $\mathrm{H} 2 \mathrm{RA}$ ) competitively bind the $\mathrm{H} 2 \mathrm{R}$, a type of G-protein coupled receptor (GPCR), ${ }^{1}$ and block the natural stimulation of its downstream signal transduction cascade by histamine; famotidine (e.g., Pepcid ${ }^{6}$ ) and ranitidine (e.g., Zantac ${ }^{\sigma}$ ) are exemplary H2RAs.

A dense web of functional linkages exists between histamine and H2RAs, on the one hand, and disparate physiological pathways on the other hand. These downstream signaling pathways include gastrointestinal systems (acid reduction) as well as the dysregulated inflammatory cascades (cytokine storm) that likely underlie much of the pathophysiology of COVID-19. ${ }^{1}$ The mechanistic basis of a putative role of famotidine in COVID-19 likely involves its roles as an H2RA versus, for instance, direct binding to the viral protease $3 \mathrm{CL}^{\text {pro }}$ (and resultant inhibition), as had been originally suspected from molecular docking studies.

Given its many possible mechanistic and regulatory linkages to signal transduction pathways, is famotidine beneficial in treating COVID-19, as gauged by outcomes involving either (i) infection transmissibility, (ii) disease severity indicators (e.g., likelihood of cases reaching the point of ventilation, WHO severity index), or (iii) mortality rates? This question remains unresolved, though not for lack of effort: since a pioneering report ${ }^{2}$ of positive clinical outcomes with famotidine use in COVID-19, over 10 studies have considered the potential therapeutic benefits of famotidine. As we recently reviewed, ${ }^{3}$ many of these reports concluded in favor of famotidine use, others found little to no association between famotidine (or PPIs) and 30-day mortality, and a recent study found a negative association for both PPIs and famotidine. These independent studies have been retrospective and observational; most were cohort-based, with some as case-series (e.g., symptom tracking across longitudinal data); most evaluated inpatient cases; and most attempted to account for confounders and other biases (e.g., via propensity-score matching). Given the conflicting reports thus far, particularly the evidence suggesting a beneficial impact of famotidine on mortality and overall disease progression (e.g., mechanical ventilation), we have undertaken the new analysis reported herein.

Note that all three parallel tracks of findings - those indicating for and against famotidine, as well as neutral (i.e., no association)rest upon substantially smaller datasets than were drawn upon in the present work. Are any beneficial effects of famotidine

\author{
; https://doi.org/10.1038/s41392-021-00689-y
}

detectable on population-wide, international scales? Is it synergistic to treat with famotidine in conjunction with aspirin, a generalpurpose anti-inflammatory? Does famotidine use correlate with any measurable parameters that may serve as biomarkers, perhaps offering mechanistic clues (e.g., serum C-reactive protein [CRP] levels as a proxy for inflammation and the cytokine storm)? This work seeks to address these questions.

We began by retrieving data from the COVID-19 Research Network supplied by TriNetX, comprising $\approx 400 \mathrm{M}$ patients across 30 countries. This federated health research network supplies electronic medical records (diagnoses, procedures, medications, etc.) as aggregated counts of de-identified information. We analyzed a cohort of 22,560 COVID-19 patients taking $\mathrm{H}_{1} / \mathrm{H}_{2}$ receptor antagonists, with a special focus on 1,379 severe cases requiring respiratory support (see CONSORT flow diagram, Supplementary Fig. 1). We defined 'death' as the primary outcome, and sought to mitigate confounder bias via propensity-score matching to achieve stratified and balanced sub-cohorts across age and gender (see Supplementary Methods). A total of $n=$ 257,864 COVID-19 cases were considered; of these, (i) 7,479 died, (ii) 18,624 used famotidine, (iii) 8,335 used cetirizine, (iv) 3,928 used loratadine, (v) 23,148 used aspirin, and (vi) 5,955 used aspirin and famotidine. Measures of association, risk ratios (RRs) and odds ratios (ORs), along with their respective 95\% confidence intervals $(\mathrm{Cls})$, were calculated, as were Kaplan-Meier survival curves.

We statistically analyzed outcomes for treatment with (i) the H1RAs loratadine (e.g., Claritin ${ }^{\circ}$ ) and cetirizine (e.g., Zyrtec ${ }^{\circ}$ ), (ii) the H2RA famotidine, (iii) aspirin, and (iv) a combination of famotidine and aspirin, as shown in Table 1. For cases that reached the point of respiratory support, we found a significantly reduced fatality risk for famotidine treatment (OR 0.73, Cl 0.57-0.94; Table 1, Supplementary Files 1-4). Dual-histamine receptor blockade, concurrently targeting the $\mathrm{H}_{1}$ and $\mathrm{H}_{2}$ receptors, has been thought to improve COVID-19 clinical outcomes ${ }^{4}$; however, significant improvements were not seen in our cohorts, versus famotidine alone (OR 0.75, Cl 0.39-1.46; Supplementary Files 5-8). Notably, and perhaps unexpectedly, the combination of famotidine and aspirin (344 severe cases before matching) did exhibit a significant synergistic survival benefit (OR 0.55, Cl 0.39-0.78; Fig. 1; Supplementary Files 9-12). The RR for death decreased by $32.5 \%$-an immense benefit, given the more than 3.8 million COVID-19-related deaths thus far. Note that, because of methodological reasons related to data availability and reduced statistical power upon further stratification, we did not further group cases into sub-cohorts based on disease severity; thus, a limitation of our work stems from the distribution of such severities almost certainly being related to the efficacy of any therapeutic intervention.

Can our findings be reconciled with recent studies of famotidine in COVID-19? A case-series of 10 non-hospitalized patients found that self-administration of famotidine had 
Table 1. Statistical outcomes for patients requiring respiratory support, considering use/disuse of (i) $\mathrm{H}_{1}$ - or $\mathrm{H}_{2}$-receptor antagonists or aspirin, as well as (ii) a combination treatment with famotidine and aspirin

\begin{tabular}{|c|c|c|c|c|c|}
\hline $\begin{array}{l}\text { Drug compound }\left[\mathrm{H}_{1} \text { or } \mathrm{H}_{2}\right. \\
\text { antagonist] }\end{array}$ & $\begin{array}{l}\text { Number of patients in cohort } \\
\text { (after matching) }\end{array}$ & Outcome: Death & Odds ratio (OR) & $\begin{array}{l}\text { Confidence interval } \\
\text { (Cl 95\%) }\end{array}$ & Hazard ratio (HR) \\
\hline Loratadine $\left[\mathrm{H}_{1}\right]$ & 88 & 29 & 1.00 & $0.55-1.87$ & 0.84 \\
\hline Famotidine $\left[\mathrm{H}_{2}\right]$ & 563 & 161 & 0.73 & $0.57-0.94$ & 0.75 \\
\hline Aspirin (Asp) & 527 & 165 & 0.79 & $0.61-1.02$ & 0.71 \\
\hline Famotidine + Asp & 305 & 83 & 0.55 & $0.39-0.78$ & 0.53 \\
\hline
\end{tabular}

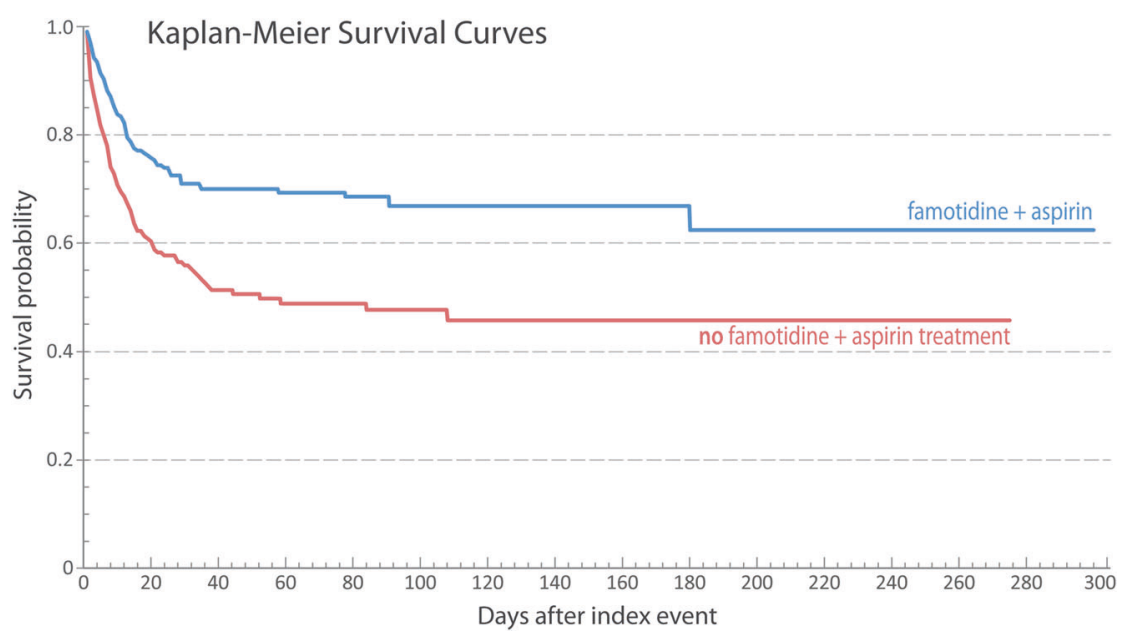

Fig. 1 Kaplan-Meier survival curves are shown for COVID-19 patients with (blue) or without (red) the dual combination treatment of famotidine and aspirin; the time-evolution of survival probabilities is given in terms of number of days after the index event (a positive COVID19 diagnosis)

uniformly beneficial impact on disease trajectories, based on quantitative symptom tracking across longitudinal data. ${ }^{3}$ Retrospective, single-center studies also found promising results, e.g. reduced risk of clinical deterioration (intubation and death) for famotidine usage in 83 and 84 hospitalized COVID-19 patients, corresponding to 9.5 and $5.1 \%$ of the analyzed cohorts, respectively. Notably, these past studies $^{3}$ found lower levels of serum markers for severe disease (e.g., ferritin, CRP [see Supp Table 1], procalcitonin) in famotidine groups, consistent with our findings and with a potential role for this H2RA in attenuating cytokine release. Finally, a new systematic review and analysis (of published reports) suggests that famotidine may be beneficial, while two other recent meta-analyses are either neutral or (statistically) inconclusive. ${ }^{3}$

If indeed famotidine is beneficial in a significant share of COVID19 cases, we suspect this could be because of the capacity of H2RAs to attenuate the pro-inflammatory pathways that become dysregulated upon infection (cytokine storms activate pro-fibrotic pathways; lung damage eventually results). Thus, a role for famotidine in COVID-19 may stem from cellular mechanisms and signaling pathways quite unrelated to its classic therapeutic role in gastroenterology - that, in turn, is an important lesson as regards drug repurposing (from a systems pharmacology perspective), targeted therapeutics, and the general idea of a COVID-19 'disease map'. ${ }^{5}$

As SARS-CoV-2 infection rates continue surging worldwide, we desperately need more data on potential therapies. The large, international, multi-center retrospective study reported here, sampling over 250,000 COVID-19 cases, hopefully helps clarify the potential benefit of clinically approved histamine antagonists such as famotidine. We anticipate that at least three prospective, randomized, controlled clinical trials that have been underway (NCT04504240, NCT04370262, and NCT04545008) will illuminate famotidine's potential therapeutic profile. Given the findings reported here, alongside the cost-effectiveness and mild sideeffects of OTC drugs like famotidine and aspirin, we suggest that further prospective clinical trials-perhaps utilizing the aspirin combination reported here (Fig. 1)-- -are advisable.

\section{DATA AVAILABILITY}

The raw data drawn upon in this work are available from the TriNetX COVID-19 Research Network (https://trinetx.com), comprising $\approx 400 \mathrm{M}$ patients distributed across 30 countries. This platform supplies electronic medical records (diagnoses, procedures, medications, etc.) as aggregated counts of de-identified information (see Supplementary Methods). In addition, we include 12 Supplementary Data Files that provide data from intermediary stages of analysis in the TriNetX platform, such as various measures of association (famotidine and risk of ventilation, risk differences, RRs and ORs, etc.), cohort statistics, raw data for Kaplan-Meier survival curves, and so on.

\section{ACKNOWLEDGEMENTS}

The authors thank F Coluzzi (Sapienza University of Rome, Italy) for contributions to the project and feedback on the manuscript. We thank K Mills (Univ of Virginia) for invaluable support in the IRB review process. This work was supported by TRR295, KFO339, and DFG PR1562/1-1. Portions of this work were also supported by the University of Virginia School of Data Science and by NSF CAREER award MCB1350957.

\section{AUTHOR CONTRIBUTIONS}

S.P. and R.P. were involved in study conception and design; all six authors were involved in data acquisition, analysis, and interpretation; C.M., S.P., R.P., and P.E.B. 
helped draft the initial paper; R.P. and P.E.B. supervised the project; C.M., R.P., S.P., and P.E.B. critically revised the manuscript. All authors read and approved the final work.

\section{ADDITIONAL INFORMATION}

Supplementary information The online version contains supplementary material available at https://doi.org/10.1038/s41392-021-00689-y.

Patient consent for publication: Not required; see Supplementary Methods section for IRB review.

Competing interests: The authors declare no competing interests.

Cameron Mura (D) , Saskia Preissner ${ }^{2}$, Susanne Nahles ${ }^{2}$ Max Heiland ${ }^{2}$, Philip E. Bourne ${ }^{1}$ and Robert Preissner ${ }^{3}$ ${ }^{1}$ School of Data Science and Department of Biomedical Engineering, University of Virginia, Charlottesville, VA, USA; ${ }^{2}$ Department Oral and Maxillofacial Surgery, Charité-Universitätsmedizin Berlin, corporate member of Freie Universität Berlin, Humboldt-Universität zu Berlin, and Berlin Institute of Health, Berlin, Germany and ${ }^{3}$ Institute of

Physiology and Science-IT, Charité-Universitätsmedizin Berlin, corporate member of Freie Universität Berlin, Humboldt-Universität zu Berlin, and Berlin Institute of Health, Berlin, Germany These authors contributed equally: Cameron Mura, Saskia Preissner Correspondence: Cameron Mura (cmura@virginia.edu) or Saskia Preissner (Saskia.Preissner@charite.de)

\section{REFERENCES}

1. Ennis, M. \& Tiligada, K. Histamine receptors and COVID-19. Inflamm. Res. 70, 67-75 (2021).

2. Freedberg, D. E. et al. Famotidine use is associated with improved clinical outcomes in hospitalized COVID-19 patients: a propensity score matched retrospective cohort study. Gastroenterology 159, 1129-1131 e1123 (2020).

3. Mura, C., Preissner, S., Preissner, R. \& Bourne, P. E. A birds-eye (Re)view of acidsuppression drugs, COVID-19, and the highly variable literature. Preprint at https:// arxiv.org/abs/2104.12003 (2021).

4. Hogan II, R. B. et al. Dual-histamine receptor blockade with cetirizine-famotidine reduces pulmonary symptoms in COVID-19 patients. Pulm. Pharm. Ther. 63, 101942 (2020).

5. Loucera, C. et al. Drug repurposing for COVID-19 using machine learning and mechanistic models of signal transduction circuits related to SARS-CoV-2 infection. Signal Transduct. Target Ther. 5, 290 (2020). Attribution 4.0 International License, which permits use, sharing, adaptation, distribution and reproduction in any medium or format, as long as you give appropriate credit to the original author(s) and the source, provide a link to the Creative Commons license, and indicate if changes were made. The images or other third party material in this article are included in the article's Creative Commons license, unless indicated otherwise in a credit line to the material. If material is not included in the article's Creative Commons license and your intended use is not permitted by statutory regulation or exceeds the permitted use, you will need to obtain permission directly from the copyright holder. To view a copy of this license, visit http://creativecommons. org/licenses/by/4.0/.

(c) The Author(s) 2021 